\title{
Saying sorry, but not sorry
}

The most demanding art form in the business is that of replying in print to criticism of work already published. By demonstration, practitioners of all disciplines seem to have arrived at similar techniques.

Publication may not be the unalloyed benefit it is supposed to be by those observers of the research profession who repeatedly remark that a dozen publications in refereed journals count for at least one rung on almost any promotion ladder. Publication also gives hostages to fortune. Others may, for example, write in with criticisms, which is at best a nuisance and may even be a great embarrassment. Is there a recipe, other than taking great care in advance, for avoiding trouble of this kind?

One defence is to publish in journals that decline to publish comments on what may already have appeared, but this is likely to be a shrinking refuge as time goes by. The Popperian doctrine that falsification may be virtuous seems to have made a mark, even if a little off target, and the intelligence that even careful authors make mistakes appears to be spreading. Journals considering themselves immune from error and thus above the fray are a declining company. So will there be no escape from criticism?

Nobody should be dismayed. The journals that already publish comments and rejoinders (this one included) are replete with illustrations of how it is possible to reply with dignity, and all the appearence of having been in the right all along, to the most hostile and even cogent criticism. The examples in what follows have been anodysed and bowdlerized where necessary to conceal the identity of the authors and their journals. They are taken from journal issues some years back, so that passions will have cooled even when the authors recognize their own prose. That seems only fair when the practice of saying sorry without saying so is so nearly universal.

Those replying to criticisms must first choose one of the several different styles in which a rejoinder may be couched. One much favoured style is the haughty, in which the responder puts his critics firmly in their place with a patronizing generalization of the complaint.

Thus there is a complaint that runs "...this result is a trivial consequence of differential geometry...the experiments reported merely confirm...suggestions that the effect is best understood quantum mechanically are thus misleading... recent interest raised by the experiment is unwarranted." How can anybody deal with that? Quite simply, it appears. Like this:"One often encounters an ambiguity in the interpretation of a phenomenon: is it classical, or is it quantal? ... In our experiment, this ambiguity of interpretation also arises..." There follows a series of three ripostes, all slightly oblique to the complainant's arguments.

The haughty rejoinder style may naturally be elaborated as the responder's imagination will allow. It makes natural sense to remind a critic that the original objectives of the published paper were much borader, even grander, than the puny objections now raised, as with a phrase such as "We were concerned with the implications of a direct connection between .... and energetically dominated thermodynamic ensembles. This theory provides a variety of specific predictions... $X Y \& Z$ say that their experiment directly contradicts one of the predictions, but that conclusion depends on unsubstantiated assumptions."

The other most common defence against criticism is the retreat into complexity, which again is most often introduced with a few haughty sentences. Thus the last rejoinder continues with the assertion that the critics have failed to demonstrate that assumptions about the temperature of two phases are correct and that they have not allowed for changes of the mobility of certain carriers in a certain class of solids. It seems common, and no doubt effective, practice to cast aspertions on the critics' competence with references to reliance "on experiments of others" and host of similar remarks.

Haughtiness can also be combined with a defence that is the opposite of complexity - the position that the data available do not justify the intricacy of the critics' arguments, as in the sentence "We believe it would be a mistake to over-interpret the existing data." If he is but partly human, it is the critic and not the responder who will have the impression that he has made a fool of himself. The general principle (for responders) is that one should never openly say one has been mistaken.

But what if the circumstances are such as to require making a clean breast of error? Even then one can retain a sense of dignity by an appropriate choice of words. Thus, "We did not intend to rely too naively on the ... idea, though we admit that our ideas are evolving on this matter" or "We agree with these two points and will try to clarify the misunderstanding that led to the criticism, point out some problems in it and correct a concep- tual error in the analysis of the data ... which, however, has no influence on its conclusion".

The conclusion, apparently designed to exonerate everybody concerned from all imaginable errors, is that "One conclusion that should be drawn from this controversy is that theoretical models should not be tied too closely to the experimental systems, which are usually much more complex. Another comclusion is that a theoretical model may stimulate the understanding of experimental observations even though the specific assumptions on which the model is based are not fulfilled in the experiment."

Again, it seems, there is no limit to authors' ways of acknowledging they have been given pause without acknowledging anything so mundane as an error. Thus " $\mathrm{X}$ et al. raise an important point .... They then proceed to show that in the experiment of Y et al. the parameter is too large by a factor of three... We agree with their results in essence (but see below)...". This, as it turns out, is a trick for blaming the whole misunderstanding on hapless $Y$ et al., the sources of the disputed data.

Further recitation of these forms of words could only engender cynicism about the reality of the open debate about the rights and wrongs of important issues, when there are important procedural issues underlying these exchanges. The plain truth is that people in the same field as the original authors and their critics are usually quick to tell who is right and who is wrong, but that people in other fields must go to a great deal of trouble before they can find out. The result is that nobody's pride is publicly injured, and that the record is set stright among those in the know. The losers are those who read the journals for general instruction.

The remedy, curiously enough, exists already. Complaints about a published article are usually referred not merely to the original author, but to those who reviewed that publication, in the hope that they may spot a pointless misunderstanding, and who may themselves censider they have some explaining to do. There is a sense in which the eventual exchange evolves from the frequently indignant content of the complainant's first covering letter to the minuet-like formality of what eventually sees the light of day. Perhaps more unvarnished protests, and their authors' reputations, should be put directly into hazard.

John Maddox 